\title{
Magnetic Field Effects upon Neutron Star Cooling
}

\author{
M. A. Teter, D. Rilett, and S. Tsuruta \\ Department of Physics, Montana State University, Bozeman, MT \\ 59717, U.S.A.
}

\begin{abstract}
The principal mechanism for neutron-star cooling during the early period of its thermal evolution is the emission of neutrinos. Because the neutrino luminosity is very large compared to the photon luminosity, most approximations leave any small effects upon the photon luminosity during the early part of the evolution as ignorable. However, for a field strength of $H \sim 10^{12}$ gauss, taking those effects into consideration in terms of a decreased photon flux from the surface means that the energy not released from the surface as photons is still trapped within the star. Energy conservation requires that neutrino emission must make up for the difference, thereby modifying the onset of the photon era of cooling. A magnetic field of large magnitude will change the surface temperature. A proper treatment of the neutron-star cooling problem in a large magnetic field requires that tangential heat flow be taken into account. This presentation shows the preliminary results of a study that is leading toward a full two-dimensional calculation of the thermal evolution.
\end{abstract}

Our study of thermal evolution for neutron stars with magnetic fields as strong as $10^{12}$ gauss began with an emphasis upon determining the need for a full twodimensional simulation due to the directional nature of the conductivity at high magnetic field strengths. If the field has significant effects upon the evolution, it will affect the time for the star to shift from neutrino-dominated cooling to surface photon-dominated cooling. The transition time from neutrino to photon cooling is defined by the star having total neutrino and photon luminosities that are equivalent. Approximating both neutrino and photon luminosities as powers of the core temperature, $L_{\nu}=k T_{c}^{8}$ and $L_{\gamma}=a T_{c}^{b}$, the core temperature that defines the transition time is $T_{f}=(a / k)^{1 /(8-b)}$ (the parameters $k$, $a$, and $b$ are defined below). To determine the transition time, the integral of $C T \mathrm{~d} T /\left(k T^{8}+a T^{b}\right)$ is solved over the interval between the initial core temperature and the final core temperature as defined above, where $C$ is the heat capacity of the star with core temperature factored out. Since the integral is typically nonanalytic, numerical integration was used to solve it, but it represents the minimum number of parameters necessary to determine the effect of the magnetic field upon thermal evolution. All that remains is to choose values of $k, a$, and $b$, that are consistent with the model under question and constrained by Figure 25 of Tsuruta (1998). The "modified Urca" neutrino luminosity is chosen as the mechanism in all cases, giving $k=1.58 \times 10^{-33} \mathrm{erg} \mathrm{s}^{-1} \mathrm{~K}^{-8}$. The nonmagnetic photon luminosity, $L(0)$, is modified to account for the effects of the magnetic field by adopting $L_{\text {mag }}=f L(0)$. 


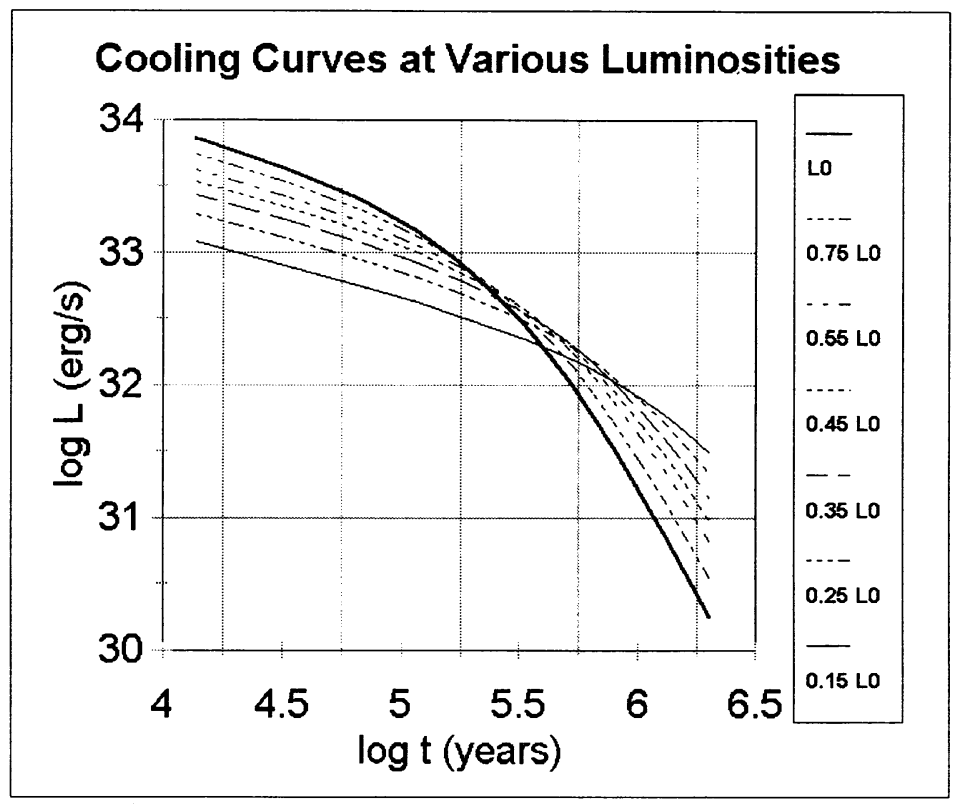

Figure 1. Cooling curves at various luminosities.

For the nonmagnetic case, the parameters are well defined within the isothermal approximation. Using the well-established nonmagnetic photon luminosity, the parameters are $a=3.3 \times 10^{11} \mathrm{erg} \mathrm{s}^{-1} K^{-2.67}$ and $b=2.67$; subsequently, the logarithm of the transition time becomes $\log t$ (years) $=4.38$, which acts as a baseline for comparison with the magnetic case.

Ideally, the magnetic case should include the use of a directional, conductive opacity for a large field (Hernquist 1984; Schaaf 1988); however, the complexity of using directional opacity would require a cumbersome, two-dimensional representation to account for tangential heat flow properly. The leading term of the radial flux in the magnetic case is $F_{r}=\lambda_{\|} \cos ^{2} \theta \partial T / \partial r$, where $\lambda_{\|}$is the conductivity along the magnetic axis. The variables $\lambda_{\|}$and $T$ are functions of angle. With luminosity being related to the average radial flux, we obtain $f<1$, thereby reducing the photon luminosity, when the field strength is $H \sim 10^{12}$ gauss. Consequently, for values of $f$ such that $0.15<f<0.75$, the logarithm of the turndown time, in years, ranges from 4.45 to 5.30 , with the latter nearly an order of magnitude different from the nonmagnetic case.

Figure 1 shows isothermal cooling curves with photon luminosity ranging over values of $f$ from 0.15 to 0.75 . A reduction of the luminosity during the neutrino-cooling era for the magnetic fields $H \lesssim 10^{13}$ gauss has profound consequences for neutron-star thermal evolution, especially in the extreme case where $f=0.15$. For instance, a significant delay in the turndown time implies the need for a nonstandard neutrino process, to be consistent with the observed data of some pulsars - definitely for PSR $1929+10$ and probably also for Geminga. How- 
ever, note that this trend (of lower photon luminosity during the neutrino-cooling era and delay of the critical switching time to the photon era) will be reversed for ultrastrong magnetic fields with $H>10^{13}$ gauss (see, e.g., Tsuruta 2000). Future work will focus upon establishing an accurate estimate of the value for $f$ and accounting for tangential heat flow in the estimate.

\section{References}

Hernquist, L. 1984, ApJS, 56, 325

Schaaf, M. E. 1988, A\&A, 205, 335

Tsuruta, S. 1998, Phys. Rep., 292, 1

Tsuruta, S. 2000, in Proc. of the Pacific Rim Conference, eds. K. S. Cheng et al. (Dordrecht: Kluwer Publ. Co.), in press 